Supporting Information

\title{
Filter-assisted Metasurface for Full-Space Wavefront Manipulation and Energy allocation
}

\author{
Chiben Zhang ${ }^{1,2}$, Xin Zhang ${ }^{3}$, Peng Xie ${ }^{1}$, Haisheng Hou ${ }^{1}$, Kaiyue Liu ${ }^{l}$, Jing Loul ${ }^{l}$, Hao Bai ${ }^{1}$, Jin \\ Zhang $^{2}$, Zhenfei $L^{2}$, Weiren Zhu*2, and Guangming Wang*l
}

1 Air and Missile Defense College, Air Force Engineering University, 710051 Xi'an, China

2 Department of Electronic Engineering, Shanghai Jiao Tong University, Shanghai 200240, China

3 China Aerospace System Simulation Technology Co., Ltd., Xi'an 710021, China

Corresponding authors: Guangming Wang (wgming01@ sina.com); Weiren Zhu

(weiren.zhu@sjtu.edu.cn)

S1. Theory and design of metasurface element

S2. Verification of photonic spin Hall effect (PSHE) for the novel metasurface.

S3. The realized gain and axis radio of patch antenna at $10 \mathrm{GHz}$.

S4. The focusing phase profiles for transmitted and reflected waves and the model of metasurface.

S5. The proportion of the two main components to the total radiated energy.

S6. The simulation for multi-vortex-beam metasurface.

S7. The angular stability of meta-atom on transmission mode and reflection mode

\section{S1 Theory and design of metasurface element}

For metasurfaces based on Pancharatnam-Berry (PB) phase theory, the amplitude and phase determine the transmission and reflection efficiencies. The EM characteristics of a meta-atom on the transmission and reflection modes can be described using two Jones matrices $R(x, y)=$ $\left(\begin{array}{ll}r_{x x} & r_{x y} \\ r_{y x} & r_{y y}\end{array}\right)$ and $T(x, y)=\left(\begin{array}{ll}t_{x x} & t_{x y} \\ t_{y x} & t_{y y}\end{array}\right)$, respectively, where $x$ and $y$ denote the two principal axes. Specially, when the meta-atom exhibits mirror symmetries the cross-polarized coefficients $r_{x y}, r_{y x}$, $t_{x y}$, and $t_{y x}$ can be regarded to be 0 . Moreover, $100 \%$ efficiency is realized when the coefficients of the PB structure satisfy the following conditions:

$$
r_{x x}=r_{y y}=0,\left|t_{x x}\right|=\left|t_{y y}\right|=1, \varphi_{x x}^{t}-\varphi_{y y}^{t}= \pm \pi(\mathrm{S} 1)
$$




$$
\left|r_{x x}\right|=\left|r_{y y}\right|=1, t_{x x}=t_{y y}=0, \varphi_{x x}^{r}-\varphi_{y y}^{r}= \pm \pi(\mathrm{S} 2)
$$

Equations (S1) and (S2) correspond to the transmission and reflection modes, respectively. For meta-atoms of transmission and reflection as an example, as shown in Figure S1(a) and S1(b).

When under LP wave illumination, we can see the all of co-polarized amplitudes, $\left|t_{x x}\right|,\left|t_{y y}\right|$, $\left|r_{x x}\right|,\left|r_{y y}\right|$, are close to 1 and corresponding phase differences in the proximity of $180^{\circ}$, as shown in Figure S1(c) and S1(d). Therefore, their efficiencies can reach nearly $100 \%$.

(a)

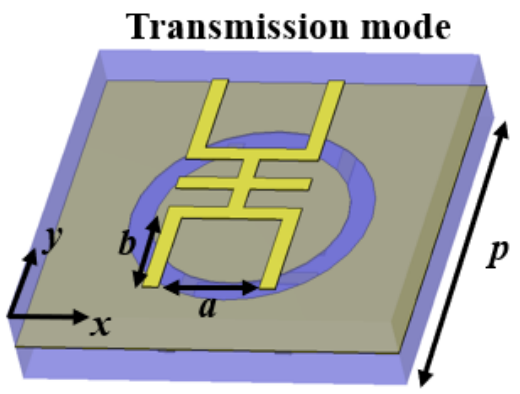

(c)

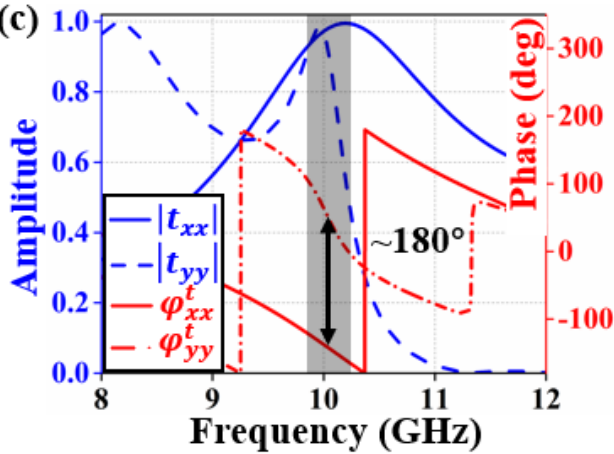

(b)
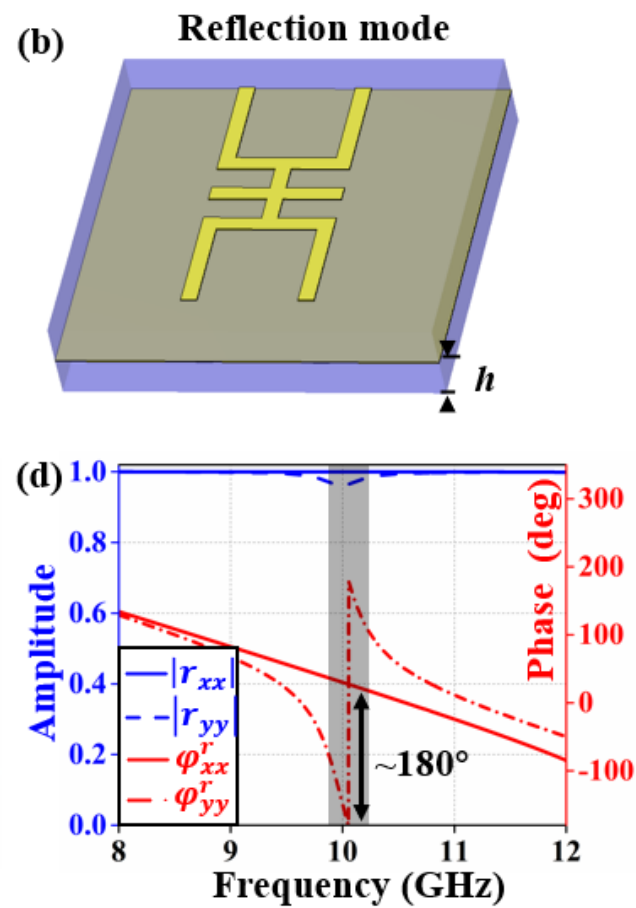

Figure S1. Topology of the transmission (a) and reflection (b) meta-atom using the composite structure and illustration of geometrical parameters. The amplitude and phase values of the transmission (c) and reflection (d) meta-atoms under LP wave illumination.

Actually, the design of PB meta-atoms on the reflection mode is easier than on the transmission mode because only the phase difference between $\varphi_{x x}^{r}$ and $\varphi_{y y}^{r}$ should be adjusted by $180^{\circ}$, similar to a half-wavelength wave plate, by optimizing the parameters of the structure, regardless of the reflection amplitudes $\left|r_{x x}\right|$ and $\left|r_{y y}\right|$ due to the ground plane. Only one type of response should be generated by a PB reflection meta-atom to achieve high-efficiency CP conversion. However, $100 \%$-efficiency transmission requires not only one response but also electric and magnetic responses to provide more freedom to tune the transmission characteristics of the meta-atom [32]. In the meta-atom, outer layers are for the top layer and the bottom layer and inner layer is for the middle layer. Here, a ring-slot filter structure is selected as the inner layer, which allows us to increase its efficiency by introducing magnetic resonance without changing the outer layers worked for electrical resonance, as displayed in Figure S2(a) and (b). Figure S2(c) and (d) show the transmission amplitude of cross-polarization, $\left|t_{-+}\right|$, increase considerably from 0.68 to 0.96 at $10 \mathrm{GHz}$ when the ring-slot filter is inserted into one-half of a 2-mm dielectric. The amplitude does not reach 1 is due to the dielectric loss. 
(a)

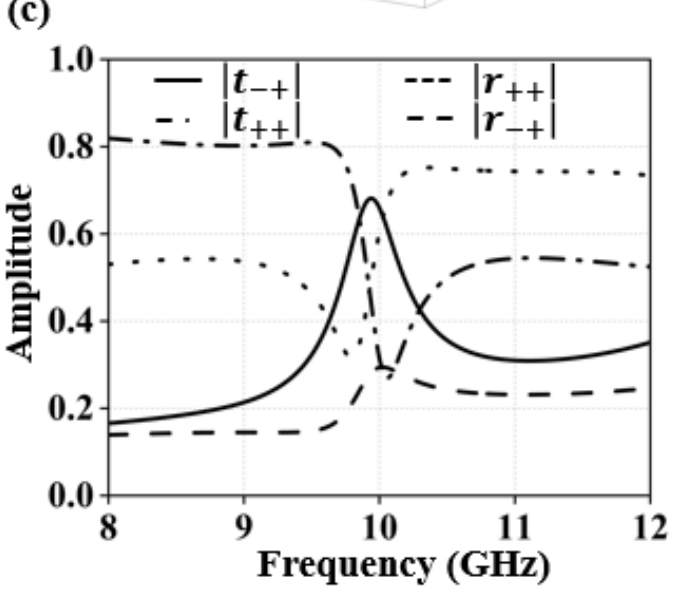

(b)
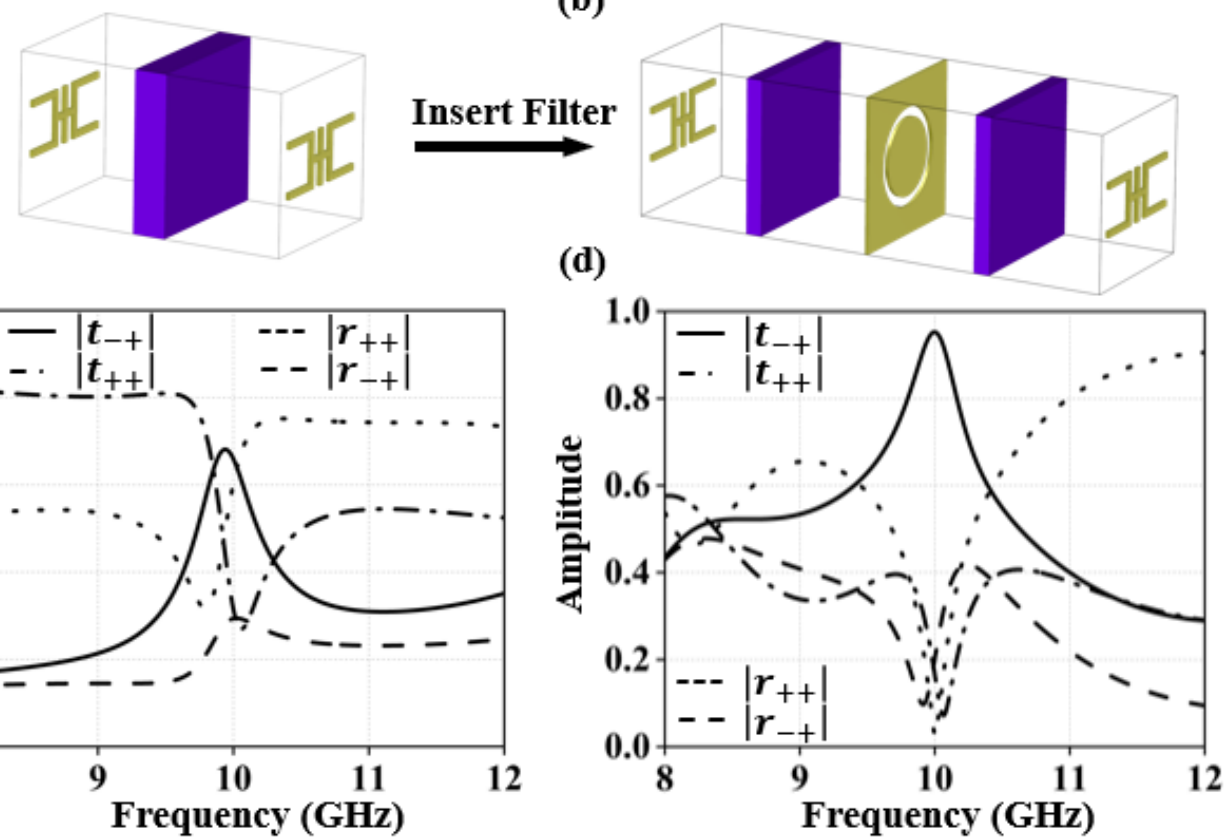

Figure S2. Topology of the transmission meta-atoms (a) without and (b) with the filter. Coefficients of reflection and transmission for meta-atoms (c) without and (d) with the filter under LHCP wave illumination. The subscripts "+" and "-" represent the CP wave with left/right handedness $\left(\sigma^{+} / \sigma^{-}\right)$ when viewed along the $+z$ direction.

Further, we found that by delicately optimizing the parameters of H-shaped structure, such as $a$ and $b$, it is possible to shift the operating frequencies of the transmission and reflection modes or make them overlap, as shown in Figure S3.
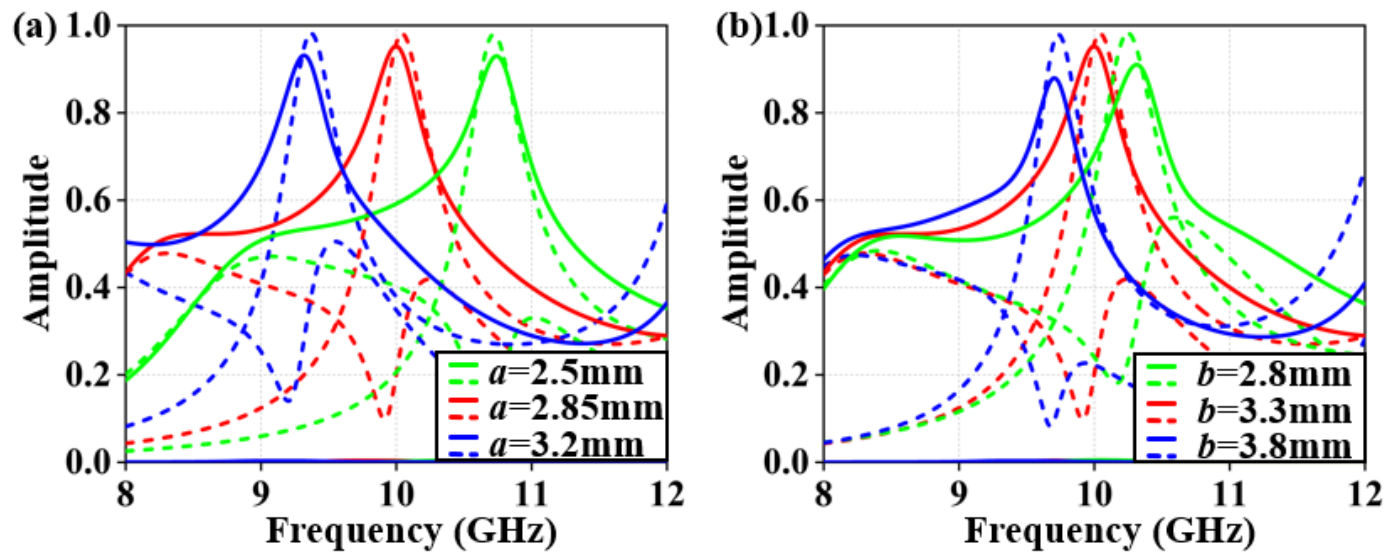

Figure S3. Simulated amplitude of transmission (solid line) and reflection (dashed line) meta-atoms vary with variations in parameter (a) $a$ and (b) $b$.

Through CST Microwave Studio we obtained the current distribution of the meta-atom with different filters to analyze the phenomenon of energy allocation under the illumination of $\mathrm{CP}$ waves on transmission, TRI and reflection mode, as displayed in Figure S4(a), (b) and (c), respectively. On the one hand, the outer H-shaped structures have the identity current distribution, which indicates that varying filter does not affect its intrinsic resonance. On the other hand, opposite currents on both sides 
of the ring slot revealed that magnetic resonance is introduced, which acts as a significant factor in the energy allocation so that the transmittance becomes progressively smaller from the transmission to the TRI and then to the reflection mode.

(a)

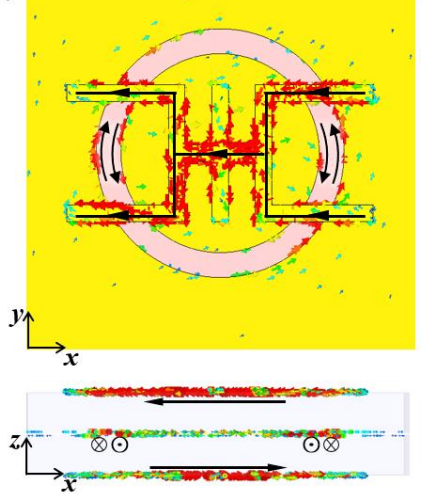

(b)

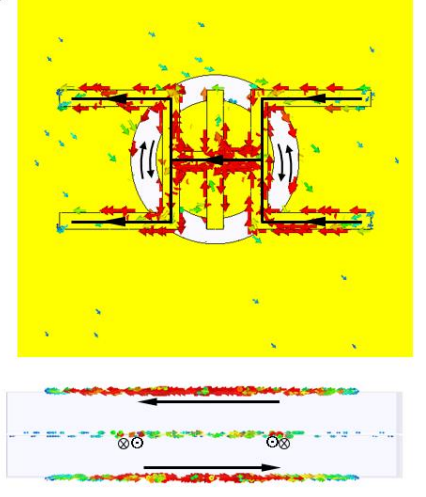

(c)

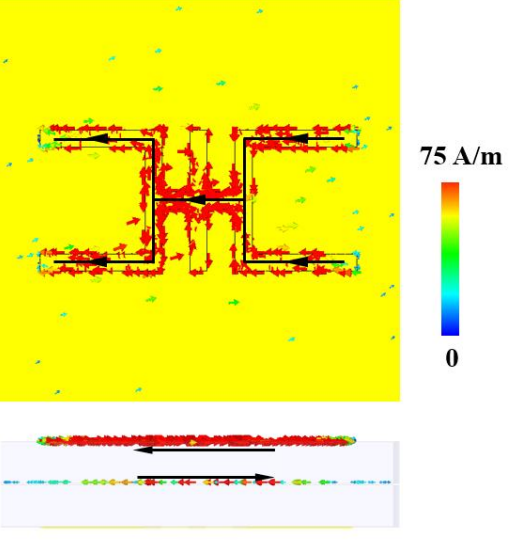

Figure S4. Current distributions of the meta-atoms under LHCP wave illumination on (a) transmission mode, (b) TRI mode and (c) reflection mode cutting at $x o y$ and $x o z$ at $10 \mathrm{GHz}$, with propagating along the $-z$ direction.

\section{S2 Verification of photonic spin Hall effect (PSHE) for the energy-allocation metasurface}

The eight meta-atoms with rotation steps of $22.5^{\circ}$ are used to construct a phase gradient supercell to validate the PSHE by simulation, as displayed in Figure S5(a). Each adjacent metaatom has a phase difference of $45^{\circ}$. For simplicity, four supercells are in a row with the open (add space) boundary in $x$-axis and $z$-axis, and periodic boundary in the $y$-axis. For example, for $\sigma+$ wave, the waves incident along the $-z$-direction are anomalously transmitted / reflected in the $x o z$ plane with angle $\left(\alpha_{t}\right) /\left(\alpha_{r}\right)$ along the $z$-axis at $10 \mathrm{GHz}$, respectively. Figure $\mathbf{S 5}(\mathbf{b})$ and (c) illustrate the PSHE with a flattened wavefront through the electric field distribution. According to the generalized Snell's laws [1], the angles can be calculated as follow:

and

$$
\begin{aligned}
& \alpha_{t}=\arcsin \left(\frac{\lambda_{0}}{2 \pi} \frac{d \varphi_{t}}{d x}\right)=+23.3^{\circ} \\
& \alpha_{r}=\arcsin \left(\frac{\lambda_{0}}{2 \pi} \frac{d \varphi_{r}}{d x}\right)=-23.3^{\circ} .
\end{aligned}
$$

Here, $\lambda_{0}$ is the free-space wavelength, $\frac{d \varphi_{t}}{d x} / \frac{d \varphi_{r}}{d x}$ is the phase gradient of transmission/ reflection, and $-z$-axis and $+z$-axis are the reference direction of the transmission and reflection modes, respectively. 
(a)
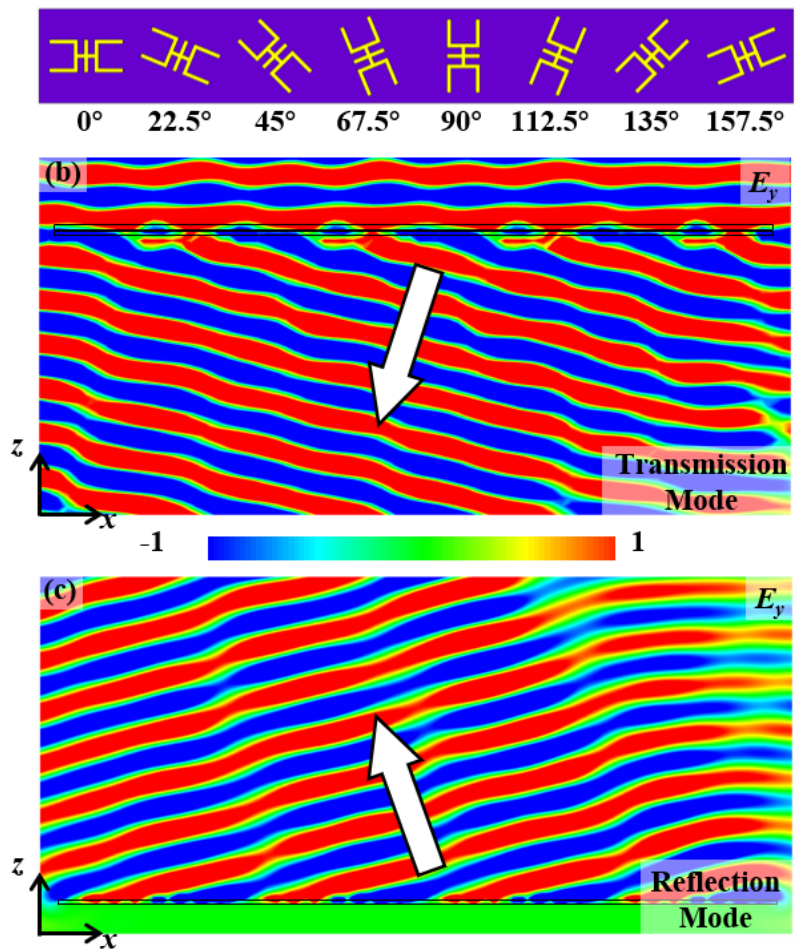

Figure S5 (a) Supercell constructed using the eight concrete elements with rotation angles $0^{\circ}, 22.5^{\circ}$, $45^{\circ}, 67.5^{\circ}, 90^{\circ}, 112.5^{\circ}, 145^{\circ}$, and $157.5^{\circ}$. The real part of the $y$-component of the electric field, $E_{y}$, on the $x o z$ plane with the phase gradient metasurfaces shined by LHCP on (b) transmission and (c) reflection modes, respectively.

The transmission and reflection angles were $+23.2^{\circ}$ and $-23.3^{\circ}$, respectively, which coincided with the theoretical calculations through the far-field patterns, as displayed in Figure S6(a) and (b). By FDTD simulation, we obtained scattered-field intensities [color map in Figure S6(c)] of transmission and reflection regions [Figure S6(d)] versus the frequency and detecting angle for the metasurface illuminated by the normally incident LHCP beams. The maximum simulated PSHE efficiency of the gradient metasurface can be calculated during the transmission mode (approximately 90.3\%) and reflection mode (approximately 96.1\%) at $10 \mathrm{GHz}$, respectively. Therefore, the gradient full-space metasurface characterizes their excellent high-efficiency wavemanipulation performance. 
(a)
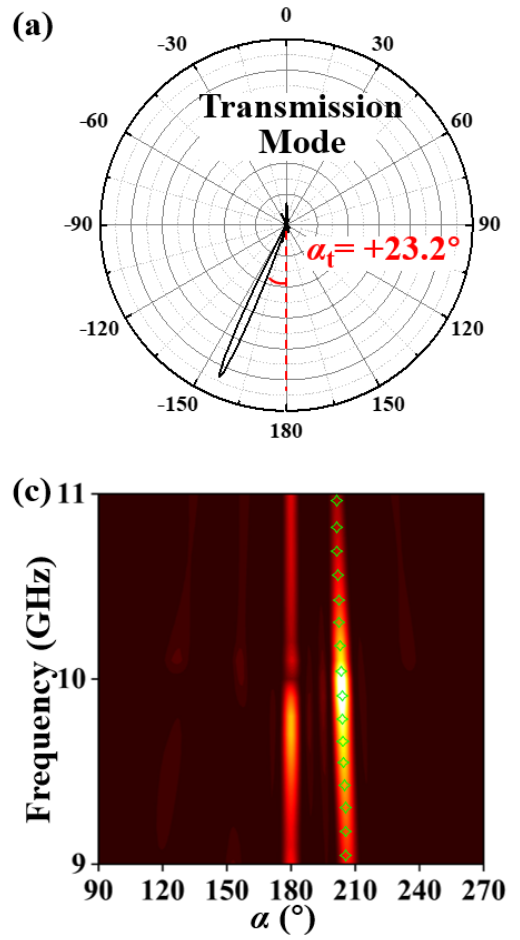

(b)

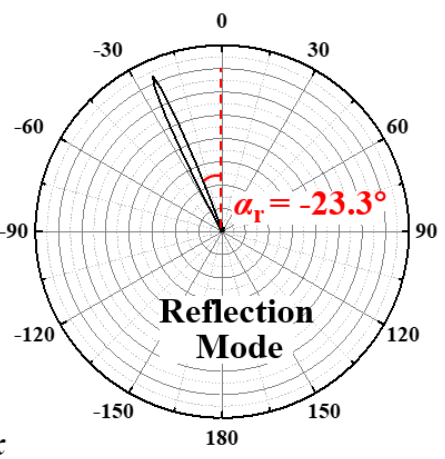

(d)

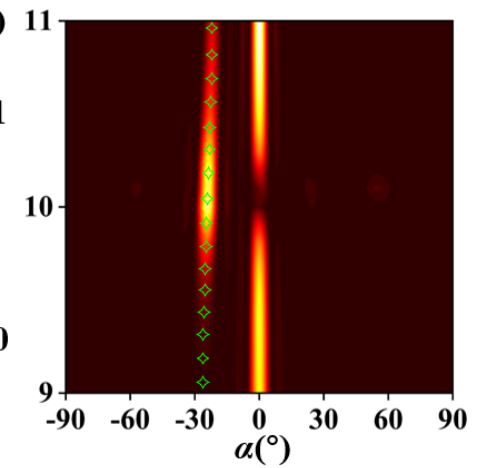

Figure S6. Two-dimensional far-field patterns (the $x o z$ plane) of the phase gradient metasurfaces excited by the $\sigma+$ wave on (a) transmission and (b) reflection modes. Scattered-field intensities on the xoz plane versus frequency under $\sigma+$ incidence on (c) transmission and (d) reflection modes. The green rhombus in (c)(d) represent the results obtained according to the Generalized Snell's law. The angle between the deflection direction and the $z$-axis is $\alpha$ and the positive direction is defined when $\alpha$ is rotated clockwise.

\section{S3. The realized gain and axis radio of patch antenna at $10 \mathrm{GHz}$.}

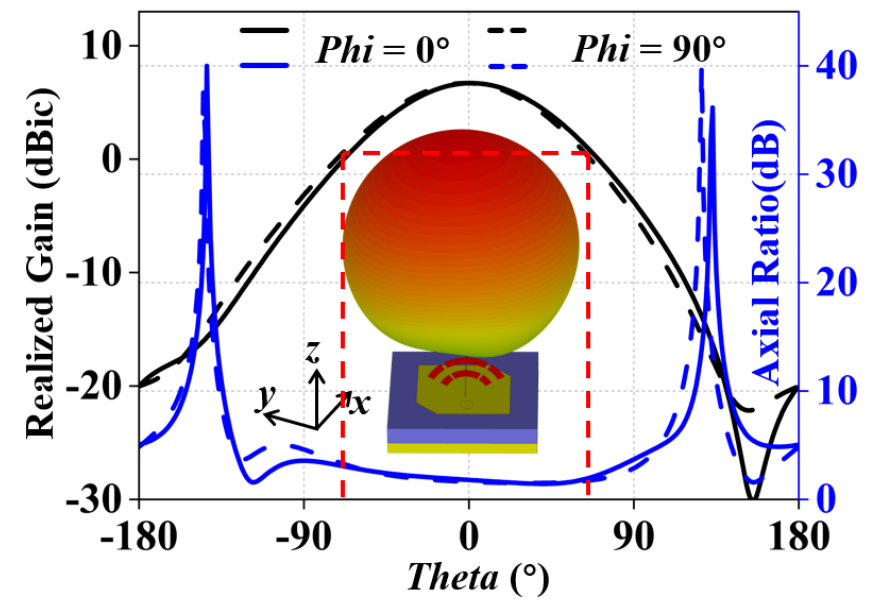

Figure S7. Radiation patterns and axial ratio (AR) of the $\sigma^{+}$patch feed at $10 \mathrm{GHz}$ when $p h i=0^{\circ}(x o z$ plane) and $p h i=90^{\circ}$ (yoz plane).

The designed patch antenna with peak gain of $6.5 \mathrm{dBic}$ and axis ratio of $1.5 \mathrm{~dB}$ at $10 \mathrm{GHz}$ both on $x o z$ and yoz plane. According two-dimensional radiation patterns, we could calculated that to 
make the edge gain less than the peak gain $6 \mathrm{~dB}(75 \%$ energy), the distance between the metasurface with the size of $123.5 \mathrm{~mm} \times 123.5 \mathrm{~mm}$ and the feed must be less than $40 \mathrm{~mm}$.

\section{S4. The focusing phase profiles for transmitted and reflected waves and the model of metasurface.}
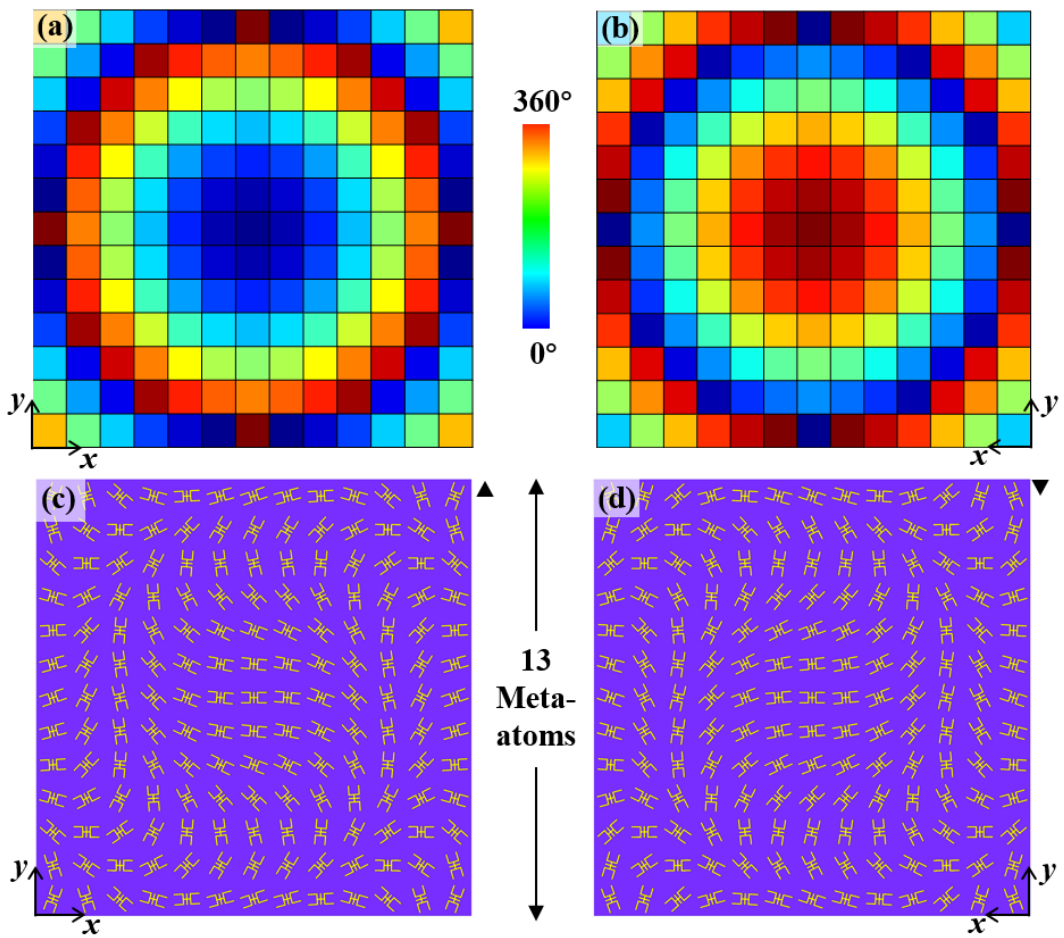

Figure S8. Phase profiles of (a) $\sigma^{+}$and (b) $\sigma$ - imparted on the both sides. The front (c) view and back (d) view of the metasurfaces.

When we place the $\sigma^{+}$patch at the focus, the metasurface with focusing phase profiles, as displayed in Figure S8, illuminate by the $\sigma+$ spherical wave along the $-z$-direction emitted crosspolarized $(\sigma-)$ plane wave in the transmission mode and co-polarized $(\sigma+)$ plane wave on the reflection mode. The phase profiles on the metasurface for $\sigma+$ and $\sigma$ - obey the following equations:

$$
\begin{gathered}
\Phi_{+}(x, y)=\frac{2 \pi}{\lambda_{0}}\left(\sqrt{x^{2}+y^{2}+F^{2}}-F\right)+\Phi_{0} \\
\Phi_{-}(x, y)=-\Phi_{+}(x, y)
\end{gathered}
$$

in which $\lambda_{0}$ corresponds to the free-space wavelength at the center frequency, $\Phi_{0}$ is the reference phase, and $F$ represents the designed focal length in Equation (S5). Equation (S6) indicates that for $\mathrm{CP}$ waves, the opposite phase distribution of the metasurface produces the same modulation of the wavefront when the spin of incident wave is reversed.

\section{S5. The proportion of the two main components to the total radiated energy}




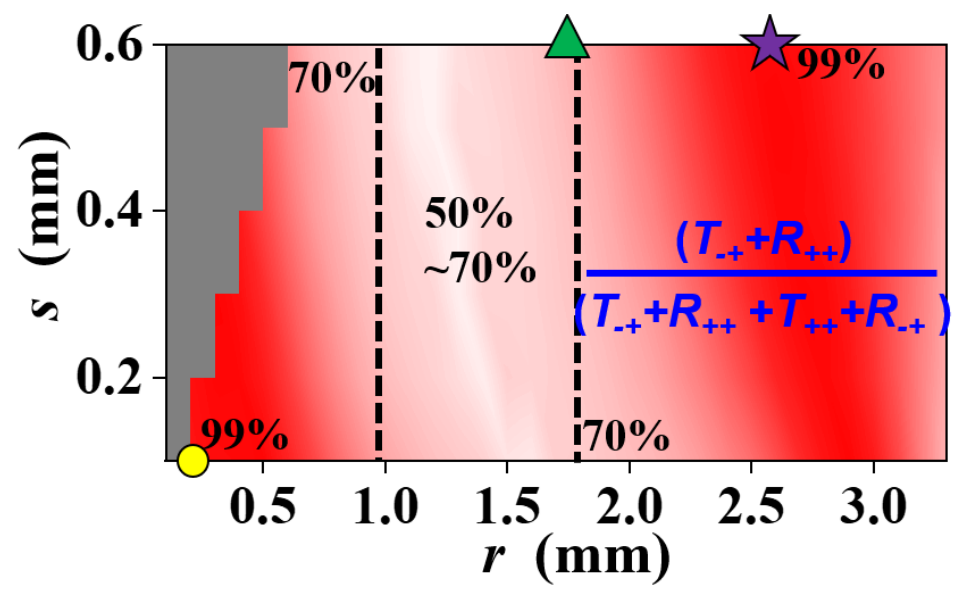

Figure S9 The proportion of the two principal components for $\mathrm{T}_{-+}$and $\mathrm{R}_{++}$to the total radiated energy.

The values of the other two components have been shown in Figures 2(d) and 2(e). It is true that a small part of the total energy is transferred to other components that we do not need in the process of allocation, but they account for a small part after all. The percentage of the two main components of the total energy varies between $70 \%$ and $99 \%$, except for the area inside the dashed line $(50 \% \sim 70 \%)$, as shown in the Figure S9. The cause of this phenomenon may be due to the reflection back and forth between multiple layers of metal, which is something we need to further improve and optimal at a later stage.

\section{S6. The simulation for multi-vortex-beam metasurface.}




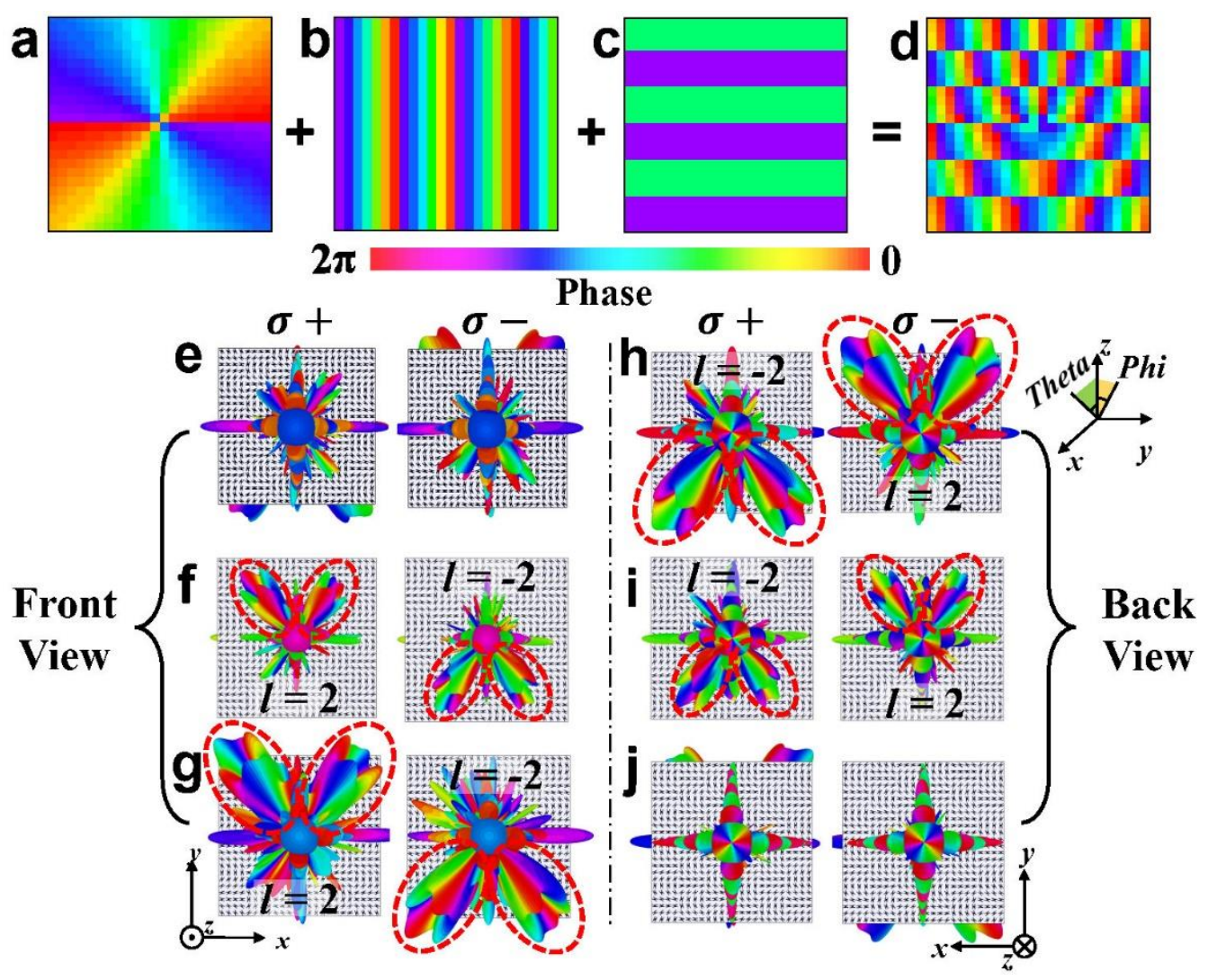

Figure S10 (a) Phase distribution of vortex beam with charge number $l= \pm 2$. (b) Phase distribution of singular deflection with an angle of phi $= \pm 30^{\circ}$. (c) Phase distribution of 1-bit coding split along with an angle of theta $= \pm 40^{\circ}$. (d) The mixed phase distribution combined by the above three for multiple vortex beam. (e)-(j) show the 3D radiation patterns of the metasurface excited by LP wave along with $z$-axis. Pictures located on the left show a front view of $\sigma+$ and $\sigma$-radiation components on the (e) transmission, (f) TRI, and (g) refection modes. As the counterpart, (h-j) pictures located on the right show the radiation in the back view.

To further verify the proposed strategy for energy-controlled wavefront manipulation in full space, another metasurface is designed that could generate eight vortex beams. The complex integrated radiation achieved by to multiplexing of three phase patterns including vortex generation, singular deflection, and coding spit, shown as Figure S10a-c. The metasurface with total phase distribution, Figure 5d, could realize precise and predictable modulation of far-field EM waves from degrees of orbital angular momentum $(l)$, deflection angle along with $x$-axis and $y$-axis. Furthermore, the energy of beams could also be allocated in full space ( $k$-vector) owing to the assist of the filter. From transmission (Figure 5e, h), to TRI (Figure 5f, i), and then to reflection (Figure 5g, h) mode, the vortex beams in reflected space (front view) gradually dominate while in transmitted space (back view) are suppressed. Specially in the TRI mode, the metasurfacce will radiate eight beams in full space with different spins and vortex modes. Four vortex beams regarding the $x o z$ plane mirror image in half space carry orbital angular momentum with opposite charge number $(l=-2$ and 2) since LP incidence wave could be detached into two waves with opposite spin ( $\sigma^{+}$and $\sigma-$ ). Similarly, the reversal of charge number will happen when $k$-vector taking negative in symmetrical space. The multi-vortex-beam metasurface breaks the limitations of space with desired energy allocation so it is expected to achieve broader applications in communications. 


\section{S7. The angular stability of meta-atom on transmission mode and reflection}

mode

(a)

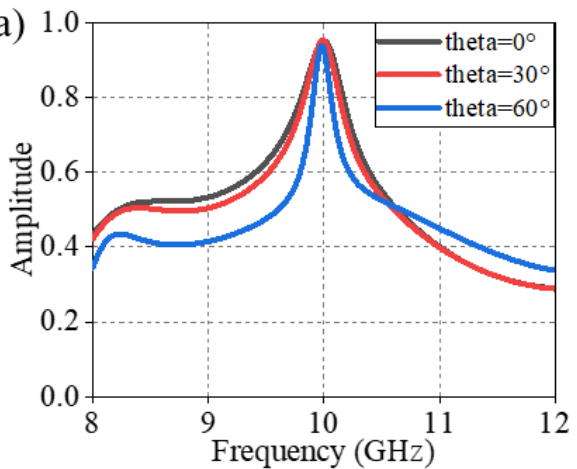

(c)

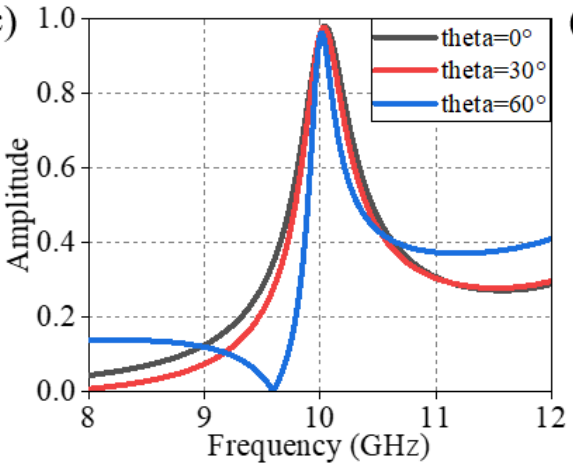

(b)

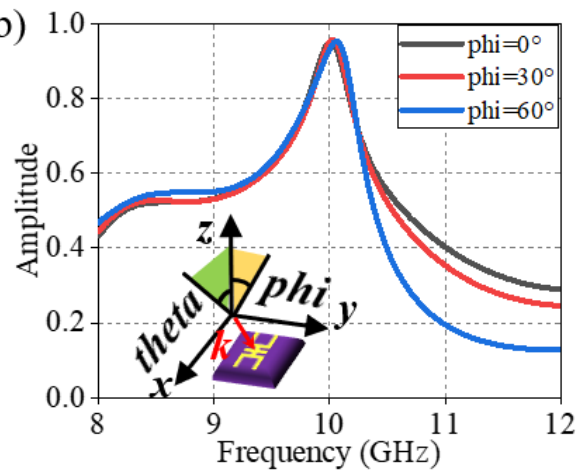

(d)

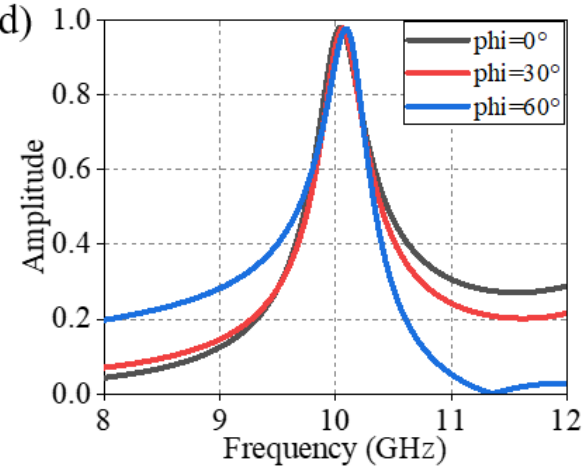

Figure S11 Angular stability of (a)(b)transmission and (c)(d)reflection meta-atoms for angle (a)(c)theta and (b)(d)phi, respectively.

By simulation, we found that the meta-atoms of reflection and transmission have good angular stability which properties can greatly reduce the error caused by spherical wave incidence, as shown in Figure S. Further, according two-dimensional radiation patterns of patch antenna, focal length $(40 \mathrm{~mm})$ and the size of the metasurface $(123.5 \mathrm{~mm} \times 123.5 \mathrm{~mm})$, we calculated that the edge gain lower than the peak gain $6 \mathrm{~dB}\left(75 \%\right.$ energy) and the maximum oblique angle is $57^{\circ}\left(<60^{\circ}\right)$. Therefore, this further weakens the effect of oblique incidence since the energy received by the metasurface decreases with increasing incidence angle.

\section{Reference}

[1] Yu, N.; Genevet, P.; Kats, M. A.; Aieta, F.; Tetienne, J.-P.; Capasso, F.; Gaburro, Z.

Light Propagation with Phase Discontinuities: Generalized Laws of Reflection and

Refraction. Science 2011, 334, 333-337 